\title{
Automatic Detection System for Defects on the Surface of Conelet Tapered Roller
}

\author{
Yang Donglin ${ }^{\mathrm{a}}$, Wu Wenlong ${ }^{\mathrm{b}}$ \\ College of Mechanical and Electronic Engineering, Changchun University of Science and \\ Technology, Changchun 130022, Jilin, China \\ aydl@cust.edu.cn, ${ }^{\mathrm{b}}$ wenlongwu@yahoo.cn
}

\begin{abstract}
Keywords: tapered roller; defect detection; Unfolding; light source; Microscopic Imaging; image
\end{abstract} collection

\begin{abstract}
Aiming at the current problems of low efficiency and high cost when manually detecting the surface flaw of tapered roller, the automatic detection system based on machine vision is developed. Combining with the features of roller detection, the principle of automatic detection and work process are introduced. The design schemes such as automatic feeding device, unfolding device of Roller and automatic sorting device are proposed. In order to obtain clear and stable roller surface image, appropriate CCD camera and optical microscope are chose and at the same, the quality of lighting source shall be improved. The experiment shows that this program can achieve the desired results and realize real-time online detection for defects on the surface of roller in the state of motion.
\end{abstract}

\section{Foreword}

In tapered roller bearings, if there are such defects on the surface as flaws and stains, it will bring serious effect on bearing quality to directly result in reducing of service life for bearing. Therefore, in industrial production, $100 \%$ defect detection shall be performed for roller surface. Currently, the detection of defects on the surface of tapered roller is still judged by manual detection method, i.e. the optometry is performed on the roller surface under the focus lamp by people. This method has such disadvantages as large labor intensity, low work efficiency, high detection error as well as great damage to the eyesight of detection personnel. The automatic detection method of defects on the surface of tapered roller is provided in this paper, aiming at the requirements of production line detection, designed special feeding and unfolding mechanism, and meanwhile by using means of digital image processing, and on the basis of geometric and texture characteristics of defects area on the surface of roller, quantificationally characterized properties of defects as well as according to defect varieties given by pattern recognition theory, realized real-time online detection for defects on the surface of roller in the state of motion.

\section{Principle of Detection}

According to the stipulation of GB/T 25767-2010, there shall not be flaws or corrosion on the surface of roller. The working surface shall be free of such defects as black rust, pitting or friction, and without burns or soft spot after acid pickling. In order to meet the real-time detection requirements of defects on the surface of roller, the entire system structure is as shown in Figure 1. The system mainly consists of feeding system (including feeding channel and drive plate), microscopic camera system, unfolding system (including friction wheel and detection device) as well as sorting system. 


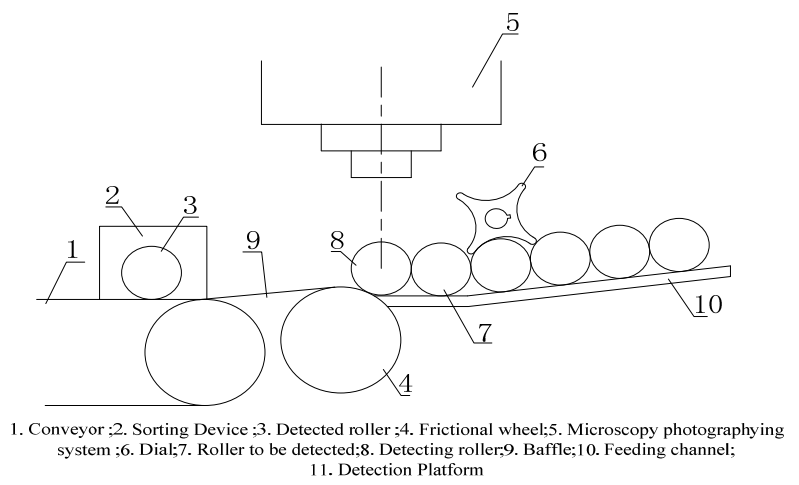

Figure 1 Diagrammatic Drawing of Detection System Structure

The basic detection process of this system is: firstly, send the roller through feed hopper to the feed channel designed on the basis of rollers from different angles, and the roller will roll down under the effect of gravity. Before the roller enters the detection area, control the conveying of roller to be detected through feeding drive plate device 6 and ensure that only one roller is sent to the detection device at a time. The roller rolls touches with friction wheel after rolling into the work area of detection device, after which the friction wheel rotates clockwise, the roller will rotate counter-clockwise under the effect of powerful friction of friction wheel to ensure that the roller unfolds its surface completely. When the roller is performing the unfolding movement, the vision system is continuously photographing the roller surface through the microscopic camera device over the roller simultaneously to capture the real-time surface image and send the collected images to the computer image processing system for recognizing, after that, the vision system will inform the sorting mechanism of recognizing results, the sorting mechanism will move under control of signal to get rid of unqualified products and safely recycle the qualified products so as to perform detection for a roller.

\section{Detection of system structure}

\section{Feeding System.}

The feeding parts are made up of the feed channel (Figure 2) and drive plate where the feeding channel A is located.

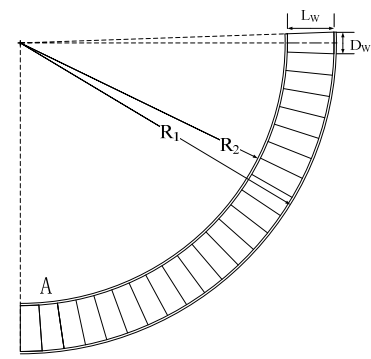

Figure 2 Diagrammatical Drawing of Feed Channel

The roller can be put in the rear part of feed channel in turn by the operator or a hopper with center rotary apparatus, this hopper can automatically arrange and send the roller to the feed channel in order by rotating itself. After entering the feed channel, the roller will roll down due to its own gravity. The track which the roller rolls is circular arc. On the basis of roller type $\left(D_{W} \times L_{W} \times 2 \varphi\right)$, the length can be calculated as follows:

$$
R_{1}=\frac{\frac{D_{W}}{2}}{\sin \phi} \approx \frac{D_{W}}{2 \bullet \phi}=\frac{90^{\circ} \bullet D_{W}}{\varphi}
$$

As this paper mainly aims at conelet tapered roller whose half cone angle is less than $2^{\circ}$, its roller length $\mathrm{L}_{\mathrm{w}}$ can be similar to roller bus length, i.e.

$$
R_{2}=R_{1}-L_{W}=\frac{90^{\circ} \bullet D_{W}}{\varphi}-L_{W}
$$


There is a drive plate at A position in Figure 2, as shown in Figure 3. When loading the material, the drive plate rotates clockwise, the shifting fork will move the roller below into the detection device, at which time the roller in the to-be-detected area of detection device will roll forward under the effect of thrust and will push out the roller that has completed the unfolding movement, which thus makes the roller to be detected enter the unfolding position, touch the friction wheel and perform unfolding movement with friction.
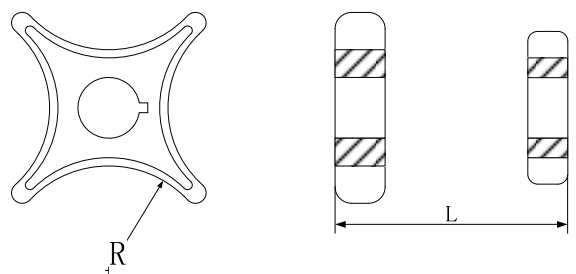

Figure 3 Diagrammatic Drawing of Drive Plate

\section{Unfolding Device of Roller.}

In the whole detection process, the first problem to be solved is about unfolding of roller. This paper adopts friction wheel unfolding mechanism, as shown in Figure 4. The roller 1 to be sent into the specific position of detection device is driven by friction wheel 3 to rotate around its own axis. If the roller surface is anticipated to unfold rapidly, the friction between the friction wheel and roller will be determined in order to ensure the unfolding effect. The friction wheel is made from rubber to increase the dynamic friction factor of unfolding wheel. The relative height of unfolding wheel and detection device can be adjusted, by properly lowering the height of unfolding wheel, the positive pressure of unfolding wheel will increase by the roller, so as to increase the friction of roller by the wheel. The detection device is made from steel plate which is polished smoothly, the roughness $\mathrm{Ra}$ is lower than 0.08 [1]. Doing works as above will effectively reduce the frictional resistance of roller by the detection device, which enables the roller to rotate when driven by the unfolding wheel.

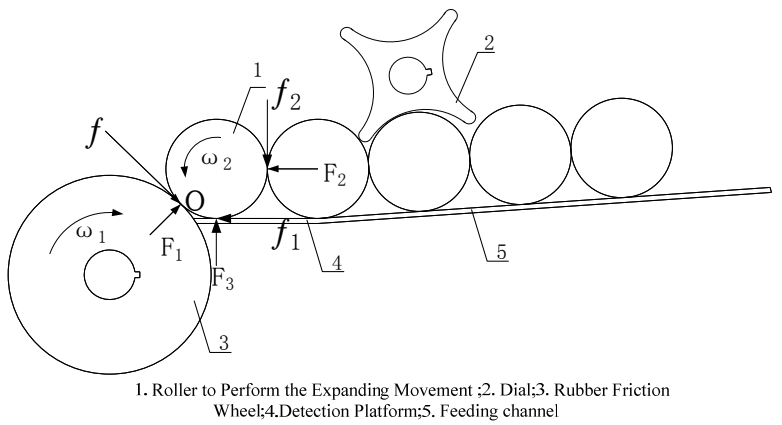

Figure 4 Diagrammatical Drawing of unfolding Device of Roller

In order to ensure the photographing effect of CCD camera above the roller, the bus on the rotating roller shall be made to keep horizontal as much as possible, so the detection device is required to design a certain angle according to half cone angle of roller, as shown in Figure 5.

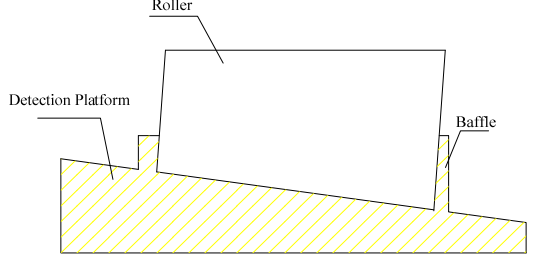

Figure 5 Diagrammatical Drawing of Detection Device

Designing of unfolding system for the roller enables the roller to perform unfolding movement rapidly and completely on the detection device. In the process of unfolding of roller, the CCD camera above continuously photographs pictures for the roller, collects pictures to the image cache through image collection card, and then determines threshold, removes the noise as well as performs gray image preprocessing such as defects image restoring, after which defects 
characteristics building and abstracting will be done for image, and comparison will be made with established defects vein database, various defects be detected by applying defects identification algorithm[2].

\section{Design of Light Source.}

While unfolding movement is being performed, defect identification shall be made for roller through machine vision system. The entire machine system includes: computer, image collection card, optical lens, CCD sensor, LED light source, $A \backslash D$ converter, as well as image processing software.

As the reflectivity of roller surface is very high, and even close to that of mirror, high reflectivity plus arc outer contour will make image lack fidelity, which thus affects detection effects. The quality of lighting source shall be improved if clear and stable roller surface image is wanted.

In order to prevent the reflected light from entering lens directly, use pointolite along the roller axis for lighting and diffuser to appropriately control the incident angle of light, so as to make the reflected light reflect to other directions by the roller surface. In general, the incident angle shall be more than $45^{\circ}$. As shown in Figure 6, the two light sources are separately placed on each side of the roller axis, the right light source lights the left half part, the left light source lights the right half part, there is certain overlapping in the middle, which can simultaneously ensure uniformity of lighting[3].
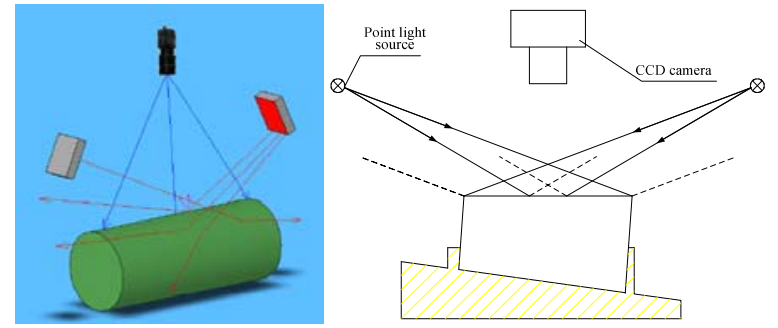

Figure 6 Lighting Scheme for Preventing Reflected Light From Entering Lens

\section{Microscopic Camera System.}

The vision system is the core part of the entire detection system. The roller diameters and lengths of common tapered roller bearings are around $10 \mathrm{~mm}$, the surface defects (such as scratch, stain) are extremely tiny, so that the roller surface images are required to enlarge through optical lens, collected and transmitted by CCD camera, and processed further by computer. As shown in block diagram 7, the roller surface image is collected by microscopic camera system consisting of microscope system and CCD camera, at which time real -time monitoring can be performed for unfolding status of roller and roller surface image effects presented by optical system in the detection cell through monitor 1 . While monitor 1 is monitoring, computer presents in real time processed image through image processing system in the monitor 2, through which the surface situation of roller can be seen clearly, the roller surface defects can be shown visually. When the monitor 2 is imaging in real time, the computer can also transmit the qualified/unqualified signal after defects identification to the sorting device, instruct which to perform sorting movement, retain and record processed image information.

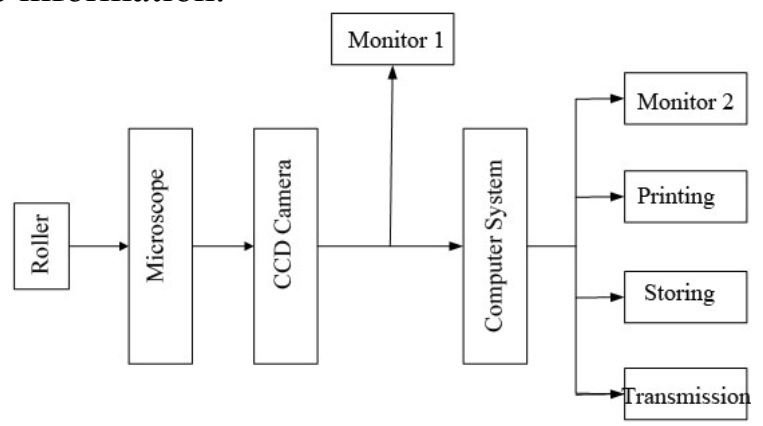

Figure 7 Block Diagram of Microscopic Imaging System

The optical microscope system adopts BXM-550 upright detection microscope manufactured by Shanghai Bingyu Optical Instrument Co. Ltd. The amplification factor of this microscope system 
can reach a range from $100 \mathrm{X}$ to $2800 \mathrm{X}$, and this microscope system is suitable for observing opaque objects. This microscope is equipped with digital interface, and can observe real-time dynamic image on the display screen of computer as well as edit, save and type pictures as required.

$1 / 2$ " black and white D130M high-speed industrial camera manufactured by Shenzhen Shangmeijie camera is adopted and combined with microscope, the maximum resolution can be up to $1280 \mathrm{H} \times 1024 \mathrm{~V}$, the pixel dimension is of $5.2 \mu \mathrm{m} \times 5.2 \mu \mathrm{m}, \mathrm{CCD}$ is progressively scanned, the exposure time is between $10 \mu$ s to $32 \mathrm{~ms}$, and such camera is featured by automatic exposure.

In order to meet the requirements of equipment design parameters to the greatest extent, the microscope system employs Wide Field 10X ocular with field diameter equal to $18 \mathrm{~mm}$ and a $40 \mathrm{X}$ object lens with NA equal to 0.6. As this system adopts the slanting for LED light source with wavelength equal to $550 \mathrm{~nm}$, according to the following formula

$$
\delta=\frac{0.5 \lambda}{N A}
$$

The minimum distance which can be resolved by this microscope is of $0.46 \mu \mathrm{m}$.

In order to enable CCD camera to make full use of resolution of object lens and obtain the minimum distance resolved by the object lens identified by CCD, the microobjective is required to be provided with large enough magnification. If the minimum distance can be resolved by CCD, i.e. the detection precision is set as $\delta^{\prime}$, the relationship between $\delta^{\prime}$ and minimum resolving distance of object lens $\delta$ shall be

$$
\delta^{\prime}=\beta \bullet \delta
$$

The magnification $\beta$ of microobjective is usually matching with NA, in terms of 40X flat-field achromatic objective lens used by this system, the magnification is often in excess of 25, the CCD detection precision will reach $11.5 \mu \mathrm{m}$ from above formula. However, in fact, the frame of microscopic system object lens is aperture stop to be usually placed on the image space focal plane of object lens to form the object space for telecentric optical path and greatly improve the measurement accuracy.

The size $\left(2 y^{\prime}\right)$ of CCD device chip usually determines the imaging range, and the relationship[4] between chip size and microscopic system object field $(2 \mathrm{y})$ is

$$
\beta=\frac{2 y^{\prime}}{2 y}
$$

Under the effect of aperture stop, $\beta$ is similar to 5 calculated from above formula, and the detection precision of entire system can reach $3 \mu \mathrm{m}$ from formula (4). If CCD subdivision technology is adopted, the detection precision can be improved further. If the subdivision is equal to 8 , the measurement precision can reach $0.375 \mu \mathrm{m}$.

\section{Sorting Device.}

When the roller on the unfolding wheel is pushed out and rolls into the sorting area, according to the signal after vision system defects identification, the sorting device will perform the removing unqualified product.

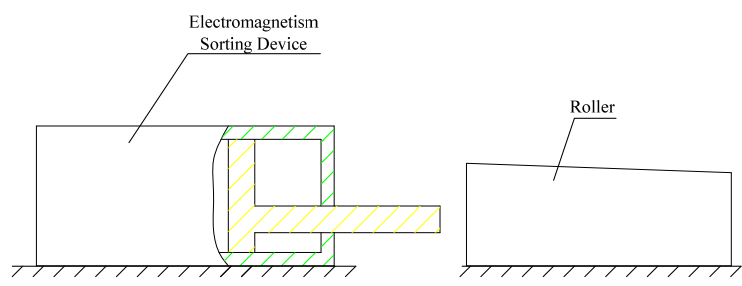

Figure 8 Diagrammatical Drawing of Electromagnetic Sorting Device

The sorting device adopts the electromagnetic principle, as shown in Figure 8, when the sensor installed in the sorting device induces that the roller reaches the designated position, it will send a confirmation signal to the computer and send the signal whether qualified or not after image defects identification to this device, if the image is identified to be unqualified, the electromagnet will cut power, the iron rod will pop out and push out the roller; if a signal indicates that the image received is qualified, the electromagnet will keep power-up state, the roller will be sent out of the detection system by the conveyor. 


\section{Conclusions}

On the basis of automatic defection system for defects on the surface of tapered roller processed by image, the design of automatic feeding and surface unfolding as a whole realizes rapid and complete unfolding of roller surface; design of light source and rotating of camera system equipment effectively lower interference of various factors with image collection; The application of computer image processing technology and pattern recognition technology realizes the automatic identification and abstraction for defects on the surface of roller; the application of electromagnetic sorting device effectively solves the problems of rapid sorting for unqualified products. This system can perform the detection with precision up to $3 \mu \mathrm{m}$ for defects on the surface of roller with a diameter between 6 and $15 \mathrm{~mm}$, and the detection speed can reach 5000 particles per hour.

\section{References}

[1] CAO Dong-hai, and LU Ze-sheng: Experiment Analysis for the Relation Between Static Coefficient of Friction and Surface Roughness, Journal of Mechanical Transmission, 2005(3): 66-68

[2] YANG Dong-lin, and YU Zheng-lin: Rapid Detection Method of Surface Defects of Steel Ball for Bearing, ACTA Armamentarii, 2009, 30(6): 797-801

[3] WANG Peng, 'Detection of Steel Surface Defect Based on Motion Vision', PhD thesis, Harbin University of Science, China, 2008:40-42

[4] WANG Qing-you: CCD application. (Tianjin University Press, China 1993.3: 161-165)

[5]MIAO Zhen-kui,WANG Feng,WANG Xiang-jun. Design of microimage analysing system[J]. Medical Equipment Journal, 1995, (5): 3-7

[6]WANG Qing-you. CCD application[M].Tianjin: Tianjin University Press, 1993.3:161-165 